\title{
A Global Convergence of Spectral Conjugate Gradient Method for Large Scale Optimization
}

\author{
Ghada M. Al-Naemi \\ Department of Mathematics, College of Computer Science and \\ Mathematics, University of Mosul, IRAQ.

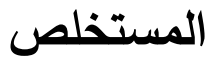

في هذا البحث, تم الاهتمام بطريقة التدرج المترافق في مسائل الامثلية غير المقيدة, وذلك

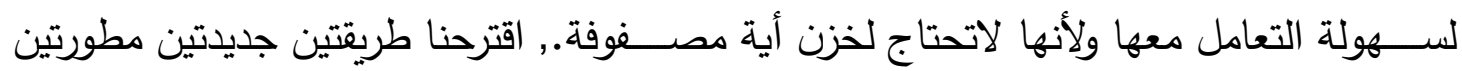
طيفيتين لطريقة (CD). الطريقتان المقترحتان تكونـان اتجاه خطوط بحث منحدرة دائما لدالة

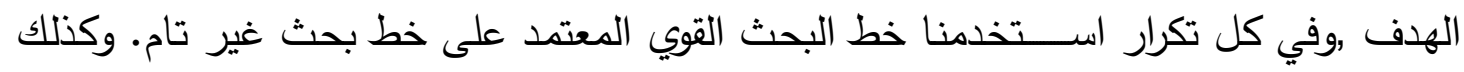
تحققان صفة التقارب الثمولي للدوال العامة غير التربيعية, النتائج العددية تظهر كفاءة الطريقتين

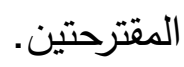

\begin{abstract}
In this paper, we are concerned with the conjugate gradient method for solving unconstrained optimization problems due to its simplicity and don't store any matrices. We proposed two spectral modifications to the conjugate descent (CD). These two proposed methods produces sufficient descent directions for the objective function at every iteration with strong Wolfe line searches and with inexact line search, and also they are globally convergent for general non-convex functions can be guaranteed. Numerical results show the efficiency of these two proposed methods.
\end{abstract}




\section{Introduction.}

Let $f: R^{n} \rightarrow R$ be continuously differentiable function. Consider the unconstrained nonlinear optimization problem:

Minimize $\mathrm{f}(\mathrm{x}), x \in R^{n}$.

We use $g(x)$ to denote to the gradient of $f$ at $x$. Due to need less computer memory especially, conjugate gradient method is very appealing for solving (1) when the number of variables is large. A conjugate gradient (CG) method generates a sequence of iterates by letting

$$
x_{k}=x_{k-1}+\alpha_{k-1} d_{k-1}, \mathrm{k}=0,1,2, \ldots
$$

where the step-length $\alpha_{k}$ is obtained by carrying out some line search, and the search direction $d_{k}$ is defined by

$$
d_{k}=\left\{\begin{array}{ll}
-g_{k}, & \text { if } k=0 \\
-g_{k}+\beta_{k} d_{k-1,}, & \text { if } k \geq 1
\end{array},\right.
$$

where $\beta_{k}$ is scalar which determines the different CG methods [11]. There are many well- known formula for $\beta_{k}$, such as the Fletcher-Reeves(FR) [7], Polak-Ribirere-Polyak (PRP) [13] and [14], Hesteness-Stiefel (HS) [10], conjugate descent (CD) [8], Liu-Story (LS) [12], and Dai-Yuan (DY) [5]. In survey paper Hager and Zhang in [9] reviewed the development of different various of nonlinear gradient methods, with especial attention given to global convergence properties.

The standard CD method proposed by Fletcher [8], specifies the $\beta_{k}^{C D}$ by

$$
\beta_{k}^{C D}=-\frac{\left\|g_{k}\right\|^{2}}{d_{k-1}^{T} g_{k-1}},
$$

where $\|$.$\| denotes the Euclidean norm of vectors. An important of the C D$ method is that the method will produce a descent direction under the strong Wolfe line search [18]

$$
\begin{aligned}
& f\left(x_{k}+\alpha_{k} d_{k}\right) \leq f\left(x_{k}\right)+\delta \alpha_{k} g_{k}^{T} d_{k}, \\
& \left|g\left(x_{k}+\alpha_{k} d_{k}\right)^{T} d_{k}\right| \leq-\sigma g_{k}^{T} d_{k},
\end{aligned}
$$

where $0<\delta<\sigma<1$. 
Another popular method to solving (1) is spectral CG method, which was developed originally by Barzilai and Browein [2]. Raydan in [17] further introduced the spectral CG method for potentially large-scale unconstrained optimization problems. Birgin and Marti'nez [3] proposed a spectral CG method by combining CG method and spectral gradient method [17], by multiplying the gradient $g_{k}$ in the second part of equation (3) by the parameter $\eta_{k}$ in the following manner:

$$
d_{k}=\left\{\begin{array}{ll}
-g_{k} & \text { if } k=0 \\
-\eta_{k} g_{k}+\beta_{k} d_{k-1}, & \text { if } k \geq 1
\end{array},\right.
$$

Zhang in [19] take FR formula and $\eta_{k}=d_{k-1}^{T} y_{k-1} /\left\|g_{k-1}\right\|^{2}$ they proved that this method can guarantee to generate descent directions and is globally convergent. Matonoha and et al in [15] proposed a modified CD method by

$$
\beta_{k}=\frac{g_{k}^{T} g_{k}}{\left|d_{k-1}^{T} g_{k-1}\right|} \text {, and } \eta_{k}=\frac{y_{k-1}^{T} d_{k-1}}{\left|d_{k-1}^{T} g_{k-1}\right|} .
$$

Zhong in [20] they proposed the spectral PRP method by using the standard PRP formula with $\eta_{k}$ defined by

$$
\eta_{k}=1-\frac{g_{k}^{T} g_{k-1} \cdot g_{k}^{T} d_{k-1}}{\left\|g_{k}\right\|^{2} \cdot d_{k-1}^{T}\left(g_{k}-g_{k-1}\right)} .
$$

Du and Liu in [6] they proposed the spectral HS method by using the standard HS formula with $\eta_{k}$ defined in (9). Liu and Jiang [11] proposed success spectral gradient method by combining the CD method and spectral gradient method by the following manner

$$
\beta_{k}=\left\{\begin{array}{l}
\beta_{k}^{C D}, \text { if } d_{k-1}^{T} g_{k} \leq 0 \\
0 \quad, \text { else }
\end{array}\right.
$$

and $\quad \eta_{k}=1-\frac{g_{k}^{T} d_{k-1}}{d_{k-1}^{T} g_{k-1}}$.

In this paper we proposed two spectral CG method, they based to the modification to the standard CD in (4), and then proposed a suitable $\eta_{k}$ for each one to get a good spectral CD-CG methods.

The rest of this paper is organized as follows. In the next section, a new modified spectral CD-CG method is proposed by combining 
modification to CD method with $\eta_{k}$ defined in (11) will be denoted by MCD1, and we give its algorithm. Section 3 will be devoted to prove the global convergence. In section 4, new proposed spectral CD-CG method is proposed by combining modification to $\mathrm{CD}$ method with $\eta_{k}$ will be defined next in this section, will be denoted by MCD2, and we give its algorithm. In section 5 will be devoted to prove the global convergence. Finally in section 6 , some numerical experiments will be done to test the efficiency of the two proposed methods.

\section{2- Modified Spectral CD Conjugate Gradient and its Algorithm (MCD1).}

In this section, we present a new modified CD method which is specified by

$$
\beta_{k}^{M C D 1}=-\frac{\left\|g_{k}\right\|^{2}}{d_{k-1}^{T} g_{k-1}}-\frac{\left\|g_{k}\right\|^{2} g_{k}^{T} d_{k-1}}{\left(d_{k-1}^{T} g_{k-1}\right)^{2}},
$$

If exact line search is used, then $\beta_{k}^{M C D 1}$ will reduce to standard $\beta_{k}^{C D}$, and $\eta_{k}$ in (11) equal one. However, we used inexact line search in our work. We put (12) with $\eta_{k}$ defined in (11) in (7), we will get the direction of our proposed method

$$
d_{k}^{M C D 1}=-\left(1-\frac{g_{k}^{T} d_{k-1}}{d_{k-1}^{T} g_{k-1}}\right) g_{k}+\left(-\frac{\left\|g_{k}\right\|^{2}}{d_{k-1}^{T} g_{k-1}}-\frac{\left\|g_{k}\right\|^{2} \cdot g_{k}^{T} d_{k-1}}{\left(d_{k-1}^{T} g_{k-1}\right)^{2}}\right) \cdot d_{k-1}
$$

\section{Algorithm (2.1) ( MCD1)}

Step 0: Given $x_{0} \in R^{n}, \varepsilon=1 * 10^{-5}, k=0, \delta \in(0,0.5)$, and $\sigma \in(\delta, 1)$.

Step 1: Set $d_{k}=-g_{k}$

Step 2: If $\left\|g_{k}\right\| \leq \varepsilon$ then stop; else continue.

Step 3: Determine the steplength $\alpha_{k}$ by using the strong Wolfe line search conditions (5) and (6).

Step 4: Calculate new point $x_{k}$ by (2).

Step 5: Compute $d_{k}^{M C D 1}$ by (13). 
Step 6: If $\mathrm{k}=\mathrm{n}$, or $\left|g_{k}^{T} g_{k-1}\right| \geq 0.2\left\|g_{k}\right\|^{2}$, set $\mathrm{k}=1$, and go to step 1; else, set $\mathrm{k}=\mathrm{k}+1$, and go to step 2 .

The following theorem shows that algorithm (2.1) possesses the sufficient descent condition with strong Wolfe line search (5) and (6).

\section{Theorem 2.1}

Let $\left\{x_{k}\right\}$ and $\left\{d_{k}\right\}$ be generated by algorithm (2.1), then we have

$$
d_{k}^{T} g_{k} \leq-c\left\|g_{k}\right\|^{2},
$$

where $c=\left(1-\sigma^{2}\right)$.

Proof: We can prove the conclusion by induction. From $\left\|g_{0}\right\|^{2}=-g_{0}^{T} d_{0}$, the conclusion (13) holds for $\mathrm{k}=0$. Now we assume that the conclusion is true for $(\mathrm{k}-1)$ and $g_{k-1} \neq 0$, i. e. $g_{k-1}^{T} d_{k-1}<0$. We need to prove that the conclusion holds for $\mathrm{k}$. Multiply both sides of (13) by $g_{k}$, we have

$$
\begin{aligned}
g_{k}^{T} d_{k} & =-\left(1-\frac{g_{k}^{T} d_{k-1}}{d_{k-1}^{T} g_{k-1}}\right)\left\|g_{k}\right\|^{2}+\left(-\frac{\left\|g_{k}\right\|^{2}}{d_{k-1}^{T} g_{k-1}}-\frac{\left\|g_{k}\right\|^{2} \cdot g_{k}^{T} d_{k-1}}{\left(d_{k-1}^{T} g_{k-1}\right)^{2}}\right) \cdot g_{k}^{T} d_{k-1} \\
& =-\left\|g_{k}\right\|^{2}-\left(\frac{g_{k}^{T} d_{k-1}}{d_{k-1}^{T} g_{k-1}}\right)^{2} \cdot\left\|g_{k}\right\|^{2} .
\end{aligned}
$$

Now, using (6) in (15), we obtain

$$
\begin{aligned}
\therefore \frac{g_{k}^{T} d_{k}}{\left\|g_{k}\right\|^{2}} & \leq-1+\left(\frac{-\sigma d_{k-1}^{T} g_{k-1}}{d_{k-1}^{T} g_{k-1}}\right)^{2} \\
& =-\left(1-\sigma^{2}\right),
\end{aligned}
$$

and since $0<\sigma<1$, we will get (14). 


\section{3- The global convergence of MCD1 method.}

In order to establish the global convergence result for the MCD1, we will impose the following assumptions for $\mathrm{f}$, which have been used often in the literature to analyze the global convergence of CG methods with inexact line search.

\section{Assumption (I): Let}

(i) the level set $\Omega=\left\{x / f(x) \leq f\left(x_{0}\right), x \in R^{n}\right\}$ is bounded.

(ii) In some neighborhood $\mathrm{N}$ of $\Omega, \mathrm{f}$ is continuously differentiable and its gradient $g$ satisfying Lipschitz conditions, namely, there exist a constant $\mathrm{L}>0$, such that $\|g(x)-g(y)\| \leq L\|x-y\|, \forall x, y \in \Omega$.

Obviously, from the Assumption (I, i) there exists a positive constant such that:

$$
B=\{\|x-y\|, \forall x, y \in \Omega\},
$$

where $\mathrm{B}$ is the diameter of $\Omega$. From Assumption (I, ii), we can also find if there exist a constant $\omega>0$, such that

$$
\left\|g_{k}\right\| \leq \omega, \forall x \in \Omega
$$

To prove global convergence by contradiction we assume that there is a positive constant $\varpi$ such that

$$
\left\|g_{k}\right\| \geq \varpi, \text { forall } k \geq 0 \text {. }
$$

We are going to prove that $\beta_{k}^{M C D 1} \geq 0$. Using (6) and (14) in (12) we will get

$$
\beta_{k}^{M C D 1} \geq(1+\sigma) \cdot \frac{1}{c}>0
$$

\section{Theorem 3.1}

Suppose that the Assumption (I) holds and consider any CG methods (2) and (7). The parameter $\beta_{k}^{M C D 1}$ defined by (12), and $\eta_{k}$ defined by (11), the 
direction $d_{k}^{M C D 1}$ is descent direction and determined $\alpha_{k}$ by using (5) and (6), if

$$
\sum_{k \geq 0} \frac{1}{\left\|d_{k}\right\|^{2}}=\infty
$$

then

$$
\operatorname{Liminf}_{k \rightarrow \infty}\left\|g_{k}\right\|=0
$$

Proof: From (12), (13) and (6) we get

$$
\begin{aligned}
\left|\beta_{k}^{M C D 1}\right| & =\left|-\frac{\left\|g_{k}\right\|^{2}}{d_{k-1}^{T} g_{k-1}}-\frac{\left\|g_{k}\right\|^{2}}{d_{k-1}^{T} g_{k-1}} g_{k}^{T} d_{k-1}\right| \\
& \leq\left|\frac{\left\|g_{k}\right\|^{2}}{d_{k-1}^{T} g_{k-1}}+\frac{\left\|g_{k}\right\|^{2}}{\left(d_{k-1}^{T} g_{k-1}\right)^{2}} \sigma d_{k-1}^{T} g_{k-1}\right| \\
& \leq\left|\frac{\left\|g_{k}\right\|^{2}}{c\left\|g_{k-1}\right\|^{2}}+\sigma \frac{\left\|g_{k}\right\|^{2}}{c\left\|g_{k-1}\right\|^{2}}\right|,
\end{aligned}
$$

Suppose that there exists a positive constants $\omega_{1}, \varpi_{1}, \omega_{2}$ and $\varpi_{2}>0$, such that $\varpi_{1} \leq\left\|g_{k}\right\| \leq \omega_{1}, \varpi_{2} \leq\left\|g_{k-1}\right\| \leq \omega_{2}$, so we get

$$
\Rightarrow\left|\beta_{k}^{M C D 1}\right| \leq \frac{|1+\sigma|}{c \varpi_{2}} \omega_{1}
$$

also,

$$
\left|\eta_{k}\right|=\left|1-\frac{g_{k}^{T} d_{k-1}}{d_{k-1}^{T} g_{k-1}}\right| \leq|1+\sigma|
$$

Take the norm of the both sides of (7) with (22) and (23), its yield

$$
\begin{aligned}
\therefore\left\|d_{k+1}\right\| & \leq\left|\eta_{k}\right|\left\|g_{k}\right\|+\left|\beta_{k}^{M C D 1}\right|\left\|d_{k-1}\right\| \\
& \leq(1+\sigma) \omega_{1}\left(1+\frac{B}{c \varpi_{2}}\right)=A
\end{aligned}
$$

This relation implies 


$$
\sum_{k \geq 1} \frac{1}{\left\|d_{k}\right\|^{2}}>\frac{1}{A^{2}} \sum_{k \geq 1} 1=+\infty .
$$

Which is contrary to proof this theorem. Therefore, the proof is complete.

Now to prove that the new algorithm is global convergence for general function, we establish a bounded for the change $\left(w_{k+1}-w_{k}\right)$ in the normalized direction $w_{k}=d_{k} /\left\|d_{k}\right\|$, which we will use to conclude, by contradiction, that the gradients cannot be bounded away from zero [16].

\section{Lemma 3.2}

Suppose that Assumption (I) hold and consider the CG algorithm (2.1), the direction $d_{k}$ given by (13) satisfies the sufficient descent condition (14), and the line search satisfying the Zoutendijk condition [21], then $d_{k} \neq 0$ and

$$
\sum_{k=1}^{\infty}\left\|w_{k}-w_{k-1}\right\|^{2}<\infty,
$$

where $w_{k}=d_{k} /\left\|d_{k}\right\|$.

Proof: Obviously, we have $d_{k} \neq 0$. Therefore, $w_{k}$ is well defined.

Now, from (19) and theorem 3.1 it follows that

$$
\sum_{k \geq 0} \frac{1}{\left\|d_{k}\right\|^{2}}<\infty,
$$

otherwise (21) holds, contradicting (19). Define

$$
u_{k}=\frac{v_{k}}{\left\|d_{k}\right\|} \text { and } r_{k}=\beta_{k}^{M C D 1} \frac{\left\|d_{k-1}\right\|}{\left\|d_{k}\right\|} \geq 0 \text {. }
$$

Therefore, we have

$$
\begin{aligned}
w_{k} & =\frac{d_{k}}{\left\|d_{k}\right\|}=\frac{-\eta_{k} g_{k}+\beta_{k}^{M C D 1} d_{k-1}}{\left\|d_{k}\right\|} \\
& =\frac{-\eta_{k} g_{k}}{\left\|d_{k}\right\|}+\beta_{k}^{M C D 1} \frac{\left\|d_{k-1}\right\|}{\left\|d_{k}\right\|} \cdot \frac{d_{k-1}}{\left\|d_{k-1}\right\|} \\
& =u_{k}+r_{k} w_{k-1} .
\end{aligned}
$$

Using the identity $\left\|w_{k}\right\|=\left\|w_{k-1}\right\|=1$, therefore 


$$
\left\|u_{k}\right\|=\left\|w_{k}-r_{k} w_{k-1}\right\|=\left\|r_{k} w_{k}-w_{k-1}\right\|
$$

(the last equality can be verified by squaring both sides). Using the condition $r_{k} \geq 0$, the triangle inequality, and (25), we obtain

$$
\begin{aligned}
\left\|w_{k}-w_{k-1}\right\| & \leq\left\|\left(1+r_{k}\right)\left(w_{k}-w_{k-1}\right)\right\| \\
& \leq\left\|w_{k}-r_{k} w_{k-1}\right\|+\left\|r_{k} w_{k}-w_{k-1}\right\| \\
& =2\left\|u_{k}\right\| .
\end{aligned}
$$

From the definition of $v_{k}$, and using (6) we get

$$
\begin{aligned}
\left\|v_{k}\right\| & =\left\|-\left(1-\frac{g_{k}^{T} d_{k-1}}{d_{k-1}^{T} g_{k-1}}\right) \cdot g_{k}\right\| \leq\left\|-(1+\sigma) g_{k}\right\| \\
& \leq(1+\sigma) \omega_{1}=E
\end{aligned}
$$

With the above estimates we get

$$
\begin{gathered}
\sum_{k \geq 1}\left\|w_{k}-w_{k-1}\right\|^{2}=\sum_{k \geq 1} 2\left\|u_{k}\right\|^{2}=4 \sum_{k \geq 1} \frac{\left\|v_{k}\right\|^{2}}{\left\|d_{k}\right\|^{2}} \\
=4 E^{2} \sum_{k \geq 1} \frac{1}{\left\|d_{k}\right\|^{2}}<\infty .
\end{gathered}
$$

Therefore (24) holds, which complete the proof.

\section{4- Modified Spectral CD Conjugate Gradient and its Algorithm (MCD2).}

In this section, we present a new modified CD method which is specified by

$$
\beta_{k}^{M C D 2}=\frac{\left\|g_{k}\right\|^{2}}{\left|d_{k-1}^{T} g_{k-1}\right|},
$$

and let us consider the new parameter $\eta_{k}$ by:

$$
\eta_{k}=\frac{\left\|g_{k}\right\|^{2}+\left|g_{k}^{T} d_{k-1}\right|}{\left|d_{k-1}^{T} g_{k-1}\right|} .
$$


If exact line search is used, then $\beta_{k}^{M C D 2}$ will reduce to standard $\beta_{k}^{C D}$, and $\eta_{k}$ equal one. However, we used inexact line search in our work. We put (26) and (27) in (7), we will get new direction

$$
d_{k}^{M C D 2}=-\left(\frac{\left\|g_{k}\right\|^{2}+\left|g_{k}^{T} d_{k-1}\right|}{\left|d_{k-1}^{T} g_{k-1}\right|}\right) g_{k}+\left(\frac{\left\|g_{k}\right\|^{2}}{\left|d_{k-1}^{T} g_{k-1}\right|}\right) \cdot d_{k-1} .
$$

\section{Algorithm (4.1) ( MCD2)}

Step 0: Given $x_{0} \in R^{n}, \varepsilon=1 * 10^{-5}, k=0, \delta \in(0,0.5)$, and $\sigma \in(\delta, 1)$.

Step 1: Set $d_{k}=-g_{k}$

Step 2: If $\left\|g_{k}\right\| \leq \varepsilon$ then stop; else continue.

Step 3: Determine the steplength $\alpha_{k}$ by using the strong Wolfe line search conditions (5) and (6).

Step 4: Calculate new point $x_{k}$ by (2).

Step 5: Compute $d_{k}^{M C D 1}$ by (28).

Step 6: If $\mathrm{k}=\mathrm{n}$, or $\left|g_{k}^{T} g_{k-1}\right| \geq 0.2\left\|g_{k}\right\|^{2}$, set $\mathrm{k}=1$, and go to step 1; else, set $\mathrm{k}=\mathrm{k}+1$, and go to step 2 .

The following theorem shows that algorithm (4.1) possesses the sufficient descent condition with strong Wolfe line search (5) and (6).

\section{Theorem 4.1}

Let $\left\{x_{k}\right\}$ and $\left\{d_{k}\right\}$ be generated by algorithm (2.1), then we have

$$
d_{k}^{T} g_{k} \leq-c\left\|g_{k}\right\|^{2},
$$

Proof: We can prove the conclusion by induction. From $\left\|g_{0}\right\|^{2}=-g_{0}^{T} d_{0}$, the conclusion (29) holds for $\mathrm{k}=0$. Now we assume that the conclusion is true 
for (k-1) and $g_{k-1} \neq 0$, i. e. $g_{k-1}^{T} d_{k-1}<0$. We need to prove that the conclusion holds for k. Multiply both sides of (28) by $g_{k}$, we have

$$
\begin{aligned}
d_{k}^{T} g_{k} & =-\left(\frac{\left\|g_{k}\right\|^{2}+\left|g_{k}^{T} d_{k-1}\right|}{\left|d_{k-1}^{T} g_{k-1}\right|}\right)\left\|g_{k}\right\|^{2}+\left(\frac{\left\|g_{k}\right\|^{2}}{\left|d_{k-1}^{T} g_{k-1}\right|}\right) \cdot g_{k}^{T} d_{k-1} \\
& =-\frac{\left\|g_{k-1}\right\|^{2}}{\left|d_{k-1}^{T} g_{k-1}\right|}\left\|g_{k}\right\|^{2}<0,
\end{aligned}
$$

let $c=\frac{\left\|g_{k-1}\right\|^{2}}{\left|d_{k-1}^{T} g_{k-1}\right|} \leq 1$, if $\left\|g_{k-1}\right\|^{2} \leq\left|d_{k-1}^{T} g_{k-1}\right|$.

W e will get (29), so the proof is complete.

\section{5- The global convergence of MCD2 method.}

In this section we are going to prove the global convergence of the proposed method MCD2.

\section{Theorem 5.1}

Suppose that the Assumption (I) holds and consider any CG methods (2) and (7). The parameter $\beta_{k}^{M C D 2}$ defined by (28), and $\eta_{k}$ defined by (27), the direction $d_{k}^{M C D 2}$ is descent direction and determined $\alpha_{k}$ by using (5) and (6), if

$$
\sum_{k \geq 0} \frac{\left\|g_{k}\right\|^{4}}{\left\|d_{k}\right\|^{2}}<\infty,
$$

then

$$
\operatorname{Liminf}_{k \rightarrow \infty}\left\|g_{k}\right\|=0 .
$$

Proof: we can rewrite (7) as follows

$$
d_{k}+\eta_{k} g_{k}=\beta_{k} d_{k-1},
$$

and squaring both side of the above equation, we get 


$$
\left\|d_{k}\right\|^{2}=\left(\frac{\left\|g_{k}\right\|^{2}}{\left|d_{k-1}^{T} g_{k-1}\right|}\right)^{2}\left\|d_{k-1}\right\|^{2}-2 \eta_{k} g_{k}^{T} d_{k}-\eta_{k}^{2}\left\|g_{k}\right\|^{2}
$$

Dividing the above equation by $\left\|g_{k}\right\|^{4}$, we have

$$
\begin{aligned}
\frac{\left\|d_{k}\right\|^{2}}{\left\|g_{k}\right\|^{4}} & =\frac{\left\|d_{k-1}\right\|^{2}}{\left(d_{k-1}^{T} g_{k-1}\right)^{2}}-\frac{\eta_{k}^{2}\left\|g_{k}\right\|^{2}+2 \eta_{k} g_{k}^{T} d_{k}}{\left\|g_{k}\right\|^{4}} \\
& \leq \frac{\left\|d_{k-1}\right\|^{2}}{\left(d_{k-1}^{T} g_{k-1}\right)^{2}}-\frac{\eta_{k}^{2}\left\|g_{k}\right\|^{2}-2 \eta_{k}\left\|g_{k}\right\|^{2}}{\left\|g_{k}\right\|^{4}} \\
& =\frac{\left\|d_{k-1}\right\|^{2}}{\left(d_{k-1}^{T} g_{k-1}\right)^{2}}-\frac{\left(\eta_{k}-1\right)^{2}}{\left\|g_{k}\right\|^{2}}+\frac{1}{\left\|g_{k}\right\|^{2}} \\
& \leq \frac{\left\|d_{k-1}\right\|^{2}}{\left(d_{k-1}^{T} g_{k-1}\right)^{2}}+\frac{1}{\left\|g_{k}\right\|^{2}} .
\end{aligned}
$$

Noting that $\left\|d_{0}\right\|^{2}=-g_{0}^{T} d_{0}=\left\|g_{0}\right\|^{2}$, we get

$$
\frac{\left\|d_{k}\right\|^{2}}{\left(g_{k}^{T} d_{k}\right)^{2}} \leq \sum_{i=0}^{k} \frac{1}{\left\|g_{i}\right\|^{2}}
$$

Therefore, it follows from (33) and (19) that

$$
\frac{\left(g_{k}^{T} d_{k}\right)^{2}}{\left\|d_{k}\right\|^{2}} \geq \frac{\varpi^{2}}{k+1},
$$

which indicates

$$
\sum_{k \geq 0} \frac{\left\|g_{k}\right\|^{4}}{\left\|d_{k}\right\|^{2}} \geq \sum_{k \geq 0} \frac{\varpi_{1}^{2}}{k+1}=+\infty .
$$

This contradicts the Zoutendijk condition [21]. Therefore the conclusion (31) holds, so the proof is complete.

The above theorem show that the new proposed method is independent to any line search is descent and global convergent. 


\section{Lemma 5.2}

Suppose that Assumption (I) hold and consider the CG algorithm (4.1), the direction $d_{k}$ given by (28) satisfies the sufficient descent condition (29), and the line search satisfying the Zoutendijk condition [21], then $d_{k} \neq 0$ and

$$
\sum_{k=1}^{\infty}\left\|w_{k}-w_{k-1}\right\|^{2}<\infty
$$

Where $w_{k}=d_{k} /\left\|d_{k}\right\|$.

Proof: Obviously, we have $d_{k} \neq 0$. Therefore, $w_{k}$ is well defined.

Now, from (19) and theorem 3.1 it follows that

$$
\sum_{k \geq 0} \frac{1}{\left\|d_{k}\right\|^{2}}<\infty
$$

otherwise (21) holds, contradicting (31). Define

$$
u_{k}=\frac{v_{k}}{\left\|d_{k}\right\|} \text { and } r_{k}=\beta_{k}^{M C D 2} \frac{\left\|d_{k-1}\right\|}{\left\|d_{k}\right\|} \geq 0 \text {. }
$$

Therefore, we have

$$
\begin{aligned}
w_{k} & =\frac{d_{k}}{\left\|d_{k}\right\|}=\frac{-\eta_{k} g_{k}+\beta_{k}^{M C D 2} d_{k-1}}{\left\|d_{k}\right\|} \\
& =\frac{-\eta_{k} g_{k}}{\left\|d_{k}\right\|}+\beta_{k}^{M C D 2} \frac{\left\|d_{k-1}\right\|}{\left\|d_{k}\right\|} \cdot \frac{d_{k-1}}{\left\|d_{k-1}\right\|} \\
& =u_{k}+r_{k} w_{k-1} .
\end{aligned}
$$

Using the identity $\left\|w_{k}\right\|=\left\|w_{k-1}\right\|=1$, therefore

$$
\left\|u_{k}\right\|=\left\|w_{k}-r_{k} w_{k-1}\right\|=\left\|r_{k} w_{k}-w_{k-1}\right\|
$$

Using the condition $r_{k} \geq 0$, the triangle inequality, and (41), we obtain

$$
\begin{aligned}
\left\|w_{k}-w_{k-1}\right\| & \leq\left\|\left(1+r_{k}\right)\left(w_{k}-w_{k-1}\right)\right\| \\
& \leq\left\|w_{k}-r_{k} w_{k-1}\right\|+\left\|r_{k} w_{k}-w_{k-1}\right\| \\
& =2\left\|u_{k}\right\| .
\end{aligned}
$$


From the definition of $v_{k}$, and using (6) we get

$$
\begin{aligned}
\left\|v_{k}\right\|= & \left\|-\left(\frac{\left\|g_{k}\right\|^{2}+\left|g_{k}^{T} d_{k-1}\right|}{\left|d_{k-1}^{T} g_{k-1}\right|}\right) \cdot g_{k}\right\| \\
& \leq(1+\sigma) \omega_{1}=Z
\end{aligned}
$$

With the above estimates we get

$$
\begin{gathered}
\sum_{k \geq 1}\left\|w_{k}-w_{k-1}\right\|^{2}=\sum_{k \geq 1} 4\left\|u_{k}\right\|^{2}=4 \sum_{k \geq 1} \frac{\left\|v_{k}\right\|^{2}}{\left\|d_{k}\right\|^{2}} \\
\leq \sum_{k \geq 1} \frac{4 Z^{2}}{\left\|g_{k}\right\|^{4}} \frac{\left\|g_{k}\right\|^{4}}{\left\|d_{k}\right\|^{2}} \\
\leq \frac{4 Z^{2}}{\varpi_{1}} \sum_{k \geq 1} \frac{\left\|g_{k}\right\|^{4}}{\left\|d_{k}\right\|^{2}}<\infty .
\end{gathered}
$$

Therefore (34) holds, which complete the proof.

\section{6- Numerical results}

In this section, we reported some numerical results that we obtained with the implementation of the two new methods MCD1 and MCD2 on a set of unconstrained test functions. The cod were written in Fortran 90 and in double precision arithmetic. Our experiments performed on a set of (35) large scale nonlinear unconstrained test functions. These test functions are contributed in CUTE (Bongratz [4] and Andrei [1]).

All these algorithms are implemented with strong Wolfe Powell line search conditions (5) and (6) with $\delta=0.001$ and $\sigma=0.9$. All these methods terminated when the following stopping criterion is satisfied:

$$
\left\|g_{k+1}\right\|<1 \times 10^{-5}
$$

We record the number of iterations denoted by (NOI), the number of function evaluations denoted by (NOF), for purpose of our comparisons. Table (1) and (2) gives a computational results of the two new methods (namely: MCD1 and MCD2) against the standard CD method with $\mathrm{n}=100$ and 10000, respectively. While Table (3) and (4) gives the percentage performance of these two proposed methods (MCD1 and MCD2) against the standard CD method taking over all the tools as $100 \%$. 
A Global Convergence of Spectral Conjugate Gradient Method for Large ...

Table (1): Comparison between MCD1; MCD2 against Standard CD with different test problems with dimension $n=100$.

\begin{tabular}{|c|c|c|c|c|c|c|}
\hline \multirow{2}{*}{$\begin{array}{c}\text { Test } \\
\text { Functions }\end{array}$} & \multicolumn{2}{|c|}{$\begin{array}{c}\text { Standard CD } \\
\text { Method }\end{array}$} & \multicolumn{2}{|c|}{$\begin{array}{l}\text { MCD1 } \\
\text { Method }\end{array}$} & \multicolumn{2}{|c|}{$\begin{array}{l}\text { MCD2 } \\
\text { Method }\end{array}$} \\
\hline & NOI & NOF & NOI & NOF & NOI & NOF \\
\hline Wood & 25 & 62 & 24 & 53 & 21 & 49 \\
\hline Dixon & 465 & 1012 & 463 & 1008 & 471 & 1029 \\
\hline Powell-3 & 21 & 45 & 20 & 43 & 19 & 41 \\
\hline Strait & 60 & 122 & 28 & 68 & 6 & 15 \\
\hline Sum & 16 & 88 & 18 & 90 & 15 & 81 \\
\hline Shallow & 13 & 32 & 13 & 33 & 10 & 25 \\
\hline Wolfe & 65 & 131 & 45 & 91 & 45 & 91 \\
\hline Cosine & 9 & 20 & 8 & 18 & 8 & 18 \\
\hline BDQRTIC & 88 & 231 & 87 & 229 & 80 & 213 \\
\hline DENSCHNB & 8 & 19 & 7 & 18 & 6 & 15 \\
\hline Dixmaana & 6 & 14 & 6 & 14 & 5 & 12 \\
\hline Dixmaanb & 5 & 13 & 5 & 13 & 5 & 13 \\
\hline Dixmaanc & 6 & 15 & 5 & 13 & 5 & 13 \\
\hline Dixmaane & 115 & 336 & 46 & 137 & 46 & 137 \\
\hline Dixmaang & 51 & 154 & 46 & 139 & 50 & 152 \\
\hline Dixmaani & 92 & 261 & 46 & 137 & 45 & 134 \\
\hline Diagonal-2 & 102 & 326 & 42 & 161 & 45 & 171 \\
\hline Diagonal-4 & 2 & 5 & 2 & 5 & 2 & 5 \\
\hline Diagonal-5 & 2 & 7 & 2 & 7 & 2 & 7 \\
\hline Diagonal-6 & 2 & 7 & 2 & 7 & 2 & 7 \\
\hline
\end{tabular}


A Global Convergence of Spectral Conjugate Gradient Method for Large ...

\begin{tabular}{|c|c|c|c|c|c|c|}
\hline Rosen & 30 & 78 & 30 & 78 & 29 & 76 \\
\hline Ex. Powell & 309 & 622 & 66 & 176 & 45 & 119 \\
\hline Ex. Beal U63 & 11 & 27 & 11 & 27 & 11 & 27 \\
\hline Ex. Block Diagonal & 24 & 50 & 22 & 46 & 20 & 42 \\
\hline Ex. Himmelbau & 19 & 150 & 15 & 128 & 15 & 125 \\
\hline Ex. Penalty & 9 & 21 & 6 & 15 & 6 & 15 \\
\hline Ex. PSC1 & 11 & 24 & 9 & 21 & 7 & 16 \\
\hline Ex. Quadratic Penalty & 10 & 22 & 7 & 16 & 7 & 16 \\
\hline Ex. Three Exponential & 32 & 67 & 9 & 21 & 7 & 17 \\
\hline OSP & 49 & 160 & 49 & 160 & 49 & 160 \\
\hline Miele & 46 & 152 & 46 & 153 & 45 & 144 \\
\hline Cubic & 80 & 173 & 15 & 43 & 15 & 43 \\
\hline NONDIA (Shanno 78) & 7 & 16 & 7 & 16 & 7 & 16 \\
\hline TRIDIA & 90 & 182 & 89 & 179 & 86 & 173 \\
\hline Scaled Quadratic & 57 & 116 & 58 & 117 & 57 & 115 \\
\hline Total & 1944 & 4760 & 1355 & 3480 & 1294 & 3332 \\
\hline
\end{tabular}

Table (2): Comparison between MCD1; MCD2 against Standard CD with different test problems with dimension $\mathrm{n}=\mathbf{1 0 0 0 0}$.

\begin{tabular}{|c|c|c|c|c|c|c|}
\hline \multirow{2}{*}{$\begin{array}{l}\text { Test } \\
\text { Functions }\end{array}$} & \multicolumn{2}{|c|}{$\begin{array}{c}\text { Standard CD } \\
\text { Method }\end{array}$} & \multicolumn{2}{|c|}{$\begin{array}{l}\text { MCD1 } \\
\text { Method }\end{array}$} & \multicolumn{2}{|c|}{$\begin{array}{l}\text { MCD2 } \\
\text { Method }\end{array}$} \\
\hline & $\mathrm{NOI}$ & NOF & $\mathrm{NOI}$ & NOF & NOI & NOF \\
\hline Wood & 31 & 76 & 29 & 69 & 26 & 59 \\
\hline Dixon & 505 & 1097 & 486 & 1064 & 498 & 1078 \\
\hline Powell-3 & 22 & 47 & 21 & 45 & 20 & 43 \\
\hline
\end{tabular}


A Global Convergence of Spectral Conjugate Gradient Method for Large ...

\begin{tabular}{|c|c|c|c|c|c|c|}
\hline Strait & 66 & 134 & 56 & 115 & 6 & 15 \\
\hline Sum & 34 & 161 & 29 & 123 & 23 & 102 \\
\hline Shallow & 14 & 35 & 17 & 53 & 10 & 25 \\
\hline Wolfe & 83 & 167 & 69 & 150 & 114 & 211 \\
\hline Cosine & 12 & 29 & 9 & 20 & 9 & 20 \\
\hline BDQRTIC & 123 & 356 & 124 & 359 & 119 & 346 \\
\hline DENSCHNB & 9 & 21 & 8 & 23 & 6 & 15 \\
\hline Dixmaana & 7 & 17 & 5 & 13 & 5 & 13 \\
\hline Dixmaanb & 6 & 16 & 5 & 14 & 5 & 14 \\
\hline Dixmaanc & 7 & 18 & 6 & 16 & 6 & 16 \\
\hline Dixmaane & 401 & 1268 & 379 & 1143 & 377 & 1130 \\
\hline Dixmaang & 406 & 1304 & 402 & 1263 & 394 & 1183 \\
\hline Dixmaani & 398 & 1312 & 378 & 1139 & 377 & 1130 \\
\hline Diagonal-2 & 503 & 1638 & 346 & 1485 & 370 & 1518 \\
\hline Diagonal-4 & 3 & 7 & 3 & 7 & 2 & 6 \\
\hline Diagonal-5 & 2 & 7 & 2 & 7 & 2 & 7 \\
\hline Diagonal-6 & 2 & 7 & 2 & 7 & 2 & 7 \\
\hline Rosen & 30 & 78 & 30 & 78 & 29 & 76 \\
\hline Ex. Powell & 521 & 1051 & 132 & 405 & 53 & 154 \\
\hline Ex. Beal U63 & 12 & 29 & 12 & 29 & 12 & 29 \\
\hline Ex. Block Diagonal & 27 & 56 & 24 & 50 & 20 & 42 \\
\hline Ex. Himmelbau & 11 & 526 & 9 & 402 & 8 & 390 \\
\hline Ex. Penalty & 6 & 22 & 6 & 22 & 6 & 22 \\
\hline Ex. PSC1 & 13 & 24 & 9 & 21 & 7 & 16 \\
\hline
\end{tabular}


A Global Convergence of Spectral Conjugate Gradient Method for Large ...

\begin{tabular}{|c|c|c|c|c|c|c|}
\hline Ex. Quadratic Penalty & 10 & 32 & 11 & 35 & 7 & 16 \\
\hline Ex. Three Exponential & 35 & 81 & 12 & 31 & 8 & 21 \\
\hline OSP & 621 & 2111 & 603 & 2002 & 551 & 1815 \\
\hline Miele & 68 & 240 & 71 & 282 & 60 & 216 \\
\hline Cubic & 94 & 199 & 24 & 70 & 16 & 45 \\
\hline NONDIA (Shanno 78) & 5 & 15 & 5 & 15 & 5 & 15 \\
\hline TRIDIA & 335 & 671 & 333 & 667 & 329 & 659 \\
\hline Scaled Quadratic & 631 & 1266 & 632 & 1269 & 626 & 1253 \\
\hline Total & 5053 & 14124 & 4289 & 12499 & 4108 & 11707 \\
\hline
\end{tabular}

Table (3): Percentage performance of the MCD1 and MCD2 methods against the standard CD method with different test problems with dimension $\mathrm{n}=\mathbf{1 0 0 0 0}$.

\begin{tabular}{|c||c||c|c|}
\hline Measurement & Standard CD & MCD1 & MCD2 \\
& Method & Method & Method \\
\hline \hline NOI & $100 \%$ & $69.70 \%$ & $66.56 \%$ \\
\hline \hline NOF & $100 \%$ & $73.11 \%$ & $70.00 \%$ \\
\hline
\end{tabular}

Table (4): Percentage performance of the MCD1 and MCD2 methods against the standard CD method with different test problems with dimension $\mathrm{n}=\mathbf{1 0 0 0 0}$.

\begin{tabular}{|c||c||c|c|}
\hline Measurement & Standard CD & MCD1 & MCD2 \\
& Method & Method & Method \\
\hline \hline NOI & $100 \%$ & $84.88 \%$ & $81.30 \%$ \\
\hline NOF & $100 \%$ & $88.49 \%$ & $82.89 \%$ \\
\hline
\end{tabular}


From Table (3) we have obtained the following results: MCD1 saves ( NOI 30.30\%), (NOF 26.89\%), and MCD2 saves (NOI 33.44\%), (NOF $30.0 \%$ ) compared with standard CD method. While from Table (4) we have obtained the following results: MCD1 saves ( NOI 15.12\%), (NOF $26.89 \%$ ), and MCD2 saves (NOI 18.70\%), (NOF 17.11\%) compared with standard CD method

\section{Reference}

[1] Andrei, N., "An unconstrained optimization test functions collection". J. of Advance Modeling and Optimization, 10:147-161 (2008).

[2] Barzilai, J. and Borwein, I.M., "Two- point step size gradient methods", IMA J. Numerical Analysis, 8:141-148 (1988).

[3] Birgin, E. and Martinez, M., A spectral conjugate gradient method for unconstrained optimization, Applied Mathematics. and optimization, 43:117-128 (2001).

[4] Bongratz, I., Conn, A. R., Gould, N. I. M. and Toint, P. L., CUTE: "constrained and unconstrained testing environments", ACM Trans. Math. Softw., 21: 123-160 (1995) .

[5] Dai, Y. H. and Yuan, Y., "Convergence properties of the conjugate descent method". Adv. Math., 25:552-562 (1996).

[6] Du, X. and Liu, J., "Global convergence of a spectral HS conjugate gradient method", Advance in Control Engineering Information Science, 15:14871492 (2011). Doi:10.1016/j.proeng.2011.08.276.

[7] Fletcher, R. and Reeves, C. M., "Function minimization by conjugate gradients", Computer Journal, 7:149-154 (1964).

[8] Fletcher, R., "Practical Methods of Optimization", $2^{\text {nd }}$ ed., A WileyInterscience Publication, John Wiley \& Sons, Inc., NY, USA (1987).

[9] Hager, W. and Zhang, H., "A survey of non-linear conjugate gradient methods", Pacific J. Optimization , 2:35-58 (2006).

[10] Hestenes, M. R., and Stiefel, E. L., "Methods of conjugate gradient for solving linear systems". J. Research Nat. Bur. Standards, 49:409-436 (1952). 
[11] Liu, J., Jiang, Y., "Global Convergence of a Spectral Conjugate Gradient Method for Unconstrained Optimization". Abstract and Applied Analysis, Hindawi Publishing Cororation, 2012:1-12 (2012).

doi:10.1155/2012/758287.

[12] Liu, D. and Story, C., "Efficient generalized conjugate gradient algorithms., part 1 : Theory, J. Optimization Theory and Applications", 69: 129-137 (1991), .

[13] Polak, E., and Ribiere, G., "Not sur la convergence de directions conjugue'e". Rev. Franaise Informant Researche Operationelle, 3e Anne'e., 16:35-43 (1969a).

[14] Polyak, B. T., (1969), "The conjugate gradient method in extreme problems". URSS Comp. Math. Phys., 9: pp.94-112 (1969b).

[15] Matonoha, C., Luksan, L. and Vlcek, J., " Computational experience with conjugate gradient methods for unconstrained optimization", Technical Report, 1038:1-17 (2008).

[16] Nocedal, J. and Glibart, J., "Global convergence properties of conjugate gradient methods for optimization", SIAM J. Optimization, 2:21-42 (1992).

[17] Raydan, M., " The Barzilai, and Borwein gradient method for the large scale unconstrained minimization problem", SIAM J. Optimization, 7:26-33 (1997).

[18] Wolfe, P., Convergence conditions for ascent methods. SIAM Review, 11:226-235 (1969).

[19] Zhang, L., Zhou, W. and Li, D. H., "Global convergence of a modified Fletcher-Reeves conjugate gradient method with Armijo-type linesearch", Numerical Mathematics, 104:561-572 (2011).

doi:10.1007/s0021-006-0028-z.

[20] Zhong, W., Zhan, L.Y. and Ya, L.W., " New spectral PRP conjugate gradient method for constrained optimization, 24:16-22 (2011).

[21] Zoutendijk, G., Nonlinear programming computational methods, In Integer and Nonlinear programming, J. Abadie (Ed.), North-Holland, Amsterdam (1970). 